\title{
Surface Characterisation of PET modified using a p-DC or HIPIMS Reactive Sputter Pre-treatment
}

\author{
S.J. Hinder ${ }^{1 *}$, M. Audronis ${ }^{2}$, V. Bellido-Gonzalez², P. Mack ${ }^{3}$, M.A. Baker ${ }^{1}$ \\ ${ }^{1}$ The Surface Analysis Laboratory, Faculty of Engineering and Physical Sciences, University of \\ Surrey, Guildford, GU2 7XH, United Kingdom \\ ${ }^{2}$ Gencoa Ltd, Physics Road, Speke, Liverpool, L24 9HP, United Kingdom \\ ${ }^{3}$ ThermoFisher Scientific, Imberhorne Lane, East Grinstead, RH19 1UB, United Kingdom.
}

\begin{abstract}
:
Surface sensitive techniques have been employed to characterize a model polymer substrate surface, poly(ethylene terephthalate) (PET), after a reactive sputter pre-treatment using magnetically enhanced $\mathrm{Cu}$ or $\mathrm{Ti}$ sputter targets in a mixed $\mathrm{Ar}-\mathrm{O}_{2}$ glow discharge plasma. The plasmas are produced using either medium frequency pulsed direct current ( $p-D C)$ or low frequency high power impulse (HIPIMS) sources. X-ray photoelectron spectroscopy (XPS), time-of-flight secondary ion mass spectrometry (ToF-SIMS) and sessile drop water contact angles were employed to investigate changes in PET surface chemistry and properties following surface modification using different $\mathrm{p}-\mathrm{DC}$ and HIPIMS process parameters.
\end{abstract}

The XPS results indicate that the chemical composition of plasma treated PET surfaces (p-DC or HIPIMS) depends strongly on the processing parameters employed such as sputter target material, magnetic array type and power supply technology. XPS results demonstrate that the sputter target material employed is of primary importance as it dictates the quantity of metal deposited/implanted into the PET surface. XPS results show that the use of a Cu target resulted in 31-35 at.\% of $\mathrm{Cu}$ incorporated into the PET surface (as $\mathrm{CuO}$ ) while the use of a Ti target resulted in only 1-4 at. \% incorporation (as $\left.\mathrm{TiO}_{2}\right)$. In the SIMS spectra and XPS depth profiles of $\mathrm{Cu}$ treated PET, indicate that the $\mathrm{CuO}$ has formed a discrete film at the surface, offering predominant or total coverage of the underlying PET. However for Ti treated PET, both PET and

\footnotetext{
*Correspondence to: Steven J. Hinder, The Surface Analysis Laboratory, Faculty of Engineering \& Physical Sciences (A1), University of Surrey, Guildford, Surrey GU2 7XH, UK. 
Ti SIMS peaks are observed and the XPS C1s peak-shape is characteristic of PET, indicating that $\mathrm{Ti}$ has not formed a discrete film, but instead $\mathrm{TiO}_{2}$ species have been incorporated, probably as an island-like distribution into the surface of the PET. The formation of CuO and $\mathrm{TiO}_{2}$ on the PET surface lead to a reduction in the contact angle compared to native PET. Hence, both p-DC and HIPIMS reactive plasma pre-treatments result in a more hydrophilic surface, promoting adhesion and offering a flexible means to introduce a wide range of surface chemistries and properties to polymeric surfaces.

\section{Keywords:}

poly(ethylene terephthalate) (PET), plasma pre-treatment, HIPIMS, XPS, SIMS, reactive sputtering, vacuum web coating.

\section{Introduction}

Vacuum web coating is an important manufacturing process that is used to produce thin film coatings on substrates in a wide variety of applications [1]. The adhesion of a thin film to a polymer web is one of the most important quality characteristics of such a coated product. Thin film adhesion to such substrates can be improved significantly by a plasma pre-treatment and such treatments are now used routinely in roll-to-roll coating systems [2-11]. Magnetically enhanced glow discharge treatments $[6-8,11,12]$ are established processes that enhance both the quality and performance of plasma processed and/or vacuum coated web products. Most notably, dual rotatable magnetrons have been successfully applied for the high rate production of nanostructured, functional, polymeric web surfaces [12]. There are a large number of application areas for vacuum web plasma pre-treatment and coated materials [1, 4-12]. Examples include food packaging, flexible electronics, thin film photovoltaic cells, optoelectronic devices, thin film batteries etc. 
Thermoplastic polymers, such as polyethylene terephthalate (PET), are widely used in vacuum web coating as substrates. However such polymers typically possess relatively poor surface properties for web coating processes. However, an increasing number of technologically important applications require high performance polymers substrates. In such cases, plasma and/or ion treatments of substrate surfaces are often employed to alter surface properties and/or chemistry so as to provide a surface with the desired properties. Treated surfaces can react chemically with deposited thin films improving adhesion by orders of magnitude. Industrial application examples where plasma/ion treatments are employed include moisture barrier films, light barriers, indium tin oxide coatings and polymer based optical devices.

Power supply technologies play an important role in both substrate pre-treatment and coating processes [13]. High Power Impulse Magnetron Sputtering (HIPIMS) [14] is a recently developed power supply technology that holds great promise to enhance the properties of certain coatings. HIPIMS processes are typically characterised by high voltage (e.g. 0.7-2 kV), high current and relatively low duty cycle (e.g. $0.5-10 \%$ ) pulses, the peak power of which can be several megawatts (e.g. 1-6 MW), resulting in high levels of metal vapour ionisation (up to $\sim 90 \%$ ). Although target voltage and current waveforms exhibited by different plasma generators might be somewhat different, resulting in different plasma and sputtered flux properties, the common features of various HIPIMS power sources are: relatively low pulse frequency (typically 50 to $1000 \mathrm{~Hz}$ ), low duty cycle (typically 0.5 to $10 \%$ ) and a pulse duration range between 10 and $1000 \mu \mathrm{s}$. To date HIPIMS has mostly been used to produce metal and ceramic coatings or plasma etch alloy substrates for hard coating applications. The presence of a substantial fraction of metal ions in HIPIMS discharges, with an energy distribution from a few tens of eV to 1-2 eV [15-17], may present new polymeric web surface engineering opportunities leading to new applications. 
In this paper we present initial results of PET web pre-treatment by magnetically enhanced glow discharge techniques powered by either medium frequency $(150 \mathrm{kHz})$ pulsed direct current $(\mathrm{p}$ DC) or low frequency $(100 \mathrm{~Hz})$ high power pulse (HIPIMS) sources. Plasma pre-treatment processes were carried out in an $\mathrm{Ar}-\mathrm{O}_{2}$ atmosphere, in balanced and unbalanced modes employing $\mathrm{Cu}$ and $\mathrm{Ti}$ targets. The pre-treated samples chemical composition and film thicknesses were comprehensively characterized using XPS, ToF-SIMS, XPS depth profiling and static drop water contact angles so as to investigate changes in the chemistry and surface properties of the PET substrate following pre-treatment employing different process parameters.

\section{Experimental Procedure}

A Gencoa Ltd (Liverpool, UK) GenLab ${ }^{T M}$ coating deposition system was employed for the experiments described here. The GenLab ${ }^{\mathrm{TM}}$, utilising a magnetron fitted with either $\mathrm{Cu}$ or $\mathrm{Ti}$ targets (both $99.5 \%$ purity), was used as the plasma treatment and coating deposition source. Normal strength balanced/unbalanced and low strength (as used for radio frequency magnetron sputtering) magnetic field configurations have been investigated. A base vacuum, better than $0.002 \mathrm{~Pa}$, was attained prior to the plasma treatment. A working pressure of $2 \mathrm{~Pa}$ was employed for all plasma treatments. A Gencoa Ltd Speedflo ${ }^{\mathrm{TM}}$ (Liverpool, UK) process controller was used to supply both $\mathrm{Ar}$ and $\mathrm{O}_{2}$ to the GenLab ${ }^{\mathrm{TM}}$. A $5 \mathrm{~kW}$ ENI RPG-50 pulsed-DC power supply and a $10 \mathrm{~kW}$ Chemfilt lonsputtering $\mathrm{AB}$ Sinex-3 HIPIMS power supply were used as plasma generators. The power applied to the targets was $\sim 500 \mathrm{~W}$ in all cases and sputtering was undertaken with the metal targets being 'fully poisoned' (oxidised) during the pre-treatment process. In pulsed-DC mode the pulse frequency was $150 \mathrm{kHz}$ and the reverse time was 2 micro seconds. In HIPIMS mode the plasma treatments were carried out employing a target pulsing frequency of $100 \mathrm{~Hz}$; the pulse-on time was 50 micro seconds. DuPont Teijin ST504 125 $\mu \mathrm{m}$ thick PET samples were mounted on a $9.5 \times 10^{-2} \mathrm{~m}$ diameter drum rotating at $1 \mathrm{rpm}$ $(\sim 0.3 \mathrm{~m} / \mathrm{min}$ tangential speed). Table 1 lists the samples produced in this study and describes the process parameters used. 
XPS spectra were acquired on a ThermoFisher Scientific (East Grinstead, UK) Theta Probe spectrometer. XPS spectra were acquired using a monochromated Al Ka X-ray source (hv = $1486.6 \mathrm{eV})$. An X-ray spot of $\sim 400 \mu \mathrm{m}$ radius was employed. Survey spectra were acquired employing a pass energy of $300 \mathrm{eV}$. High resolution, core level spectra for $\mathrm{C} 1 \mathrm{~s}, \mathrm{Cu} 2 \mathrm{p}_{3 / 2}$ and O1s were acquired with a pass energy of $50 \mathrm{eV}$. All other high resolution core level spectra were acquired with a pass energy of $80 \mathrm{eV}$. XPS depth profiles were acquired on a ThermoFisher Scientific (East Grinstead, UK) K-Alpha spectrometer. XPS depth profile data was acquired using a monochromated Al Ka X-ray source while sample etching was achieved using a Ar ion gun operated at $100 \mathrm{eV}$ and delivering $\sim 1 \mu \mathrm{A}$ of $\mathrm{Ar}^{+}$etch current. All spectra were charge referenced against the $\mathrm{C} 1 \mathrm{~s}$ peak at $285.0 \mathrm{eV}$ to correct for charging effects during acquisition. Quantitative surface chemical analyses were calculated from the high resolution, core level spectra following the removal of a non-linear (Shirley) background. The ThermoFisher Scientific Avantage software was used which incorporates the appropriate sensitivity factors and corrects for the electron energy analyser transmission function. Conversion of etch time to distance was performed by applying the 'Single Overlayer' calculation (in the Avantage software) to the recorded profile which enables the layer thickness at the metal oxide/PET interface to be determined (using the Beer-Lambert equation [20]) and the etch rate can then be calculated.

ToF-SIMS analyses were carried out on an ION-TOF GmbH (Münster, Germany) TOF.SIMS 5 spectrometer. The instrument is equipped with a reflectron type analyser and microchannel plate detector. Mass data acquisition was performed by raster scanning over a $100 \times 100 \mu \mathrm{m}^{2}$ area. $\mathrm{A} 25 \mathrm{keV} \mathrm{Bi}_{3}{ }^{+}$primary ion beam delivering $0.13 \mathrm{pA}$ of current was used. A cycle time of $100 \mu$ s was employed for mass data acquisition. Charge compensation was achieved using a low energy electron flood gun. 
Sessile drop water contact angles were measured on a Krüss (Hamburg, Germany) EasyDrop DSA100 instrument using distilled, deionised water. The contact angles are an average of 10 individual contact angle measurements.

\section{Results \& Discussion;}

In Table 1 the process parameters, XPS elemental compositions and sessile drop water contact angles for all 8 samples investigated are presented. As expected XPS shows the unmodified PET reference sample is composed of only carbon (67 at.\%) and oxygen (33 at.\%). The water contact angle of the PET reference is $74.3^{\circ}$ which is consistent with the results of others [18]. In Figure 1 the XPS C1s high resolution spectrum for the PET reference is presented along with the high resolution C1s spectra for samples S4 and S5. In Figure 1 the C1s spectra for the PET reference exhibits a distinct peak shape being composed primarily of benzyl carbons, carboxyl carbon in the ester function and a methylene carbon bonded to the ester function, such a peak shape is as expected for PET [19].

The S1, S2, S3 and S4 samples, having undergone a Cu pre-treatment, are composed of carbon (27 - 31 at.\%), oxygen (36 - 38 at.\%) and copper (31 - 35\%) with traces of nitrogen. The carbon, oxygen and copper concentrations for samples S1, S2, S3 and S4 are similar (within +/2 at.\%). The water contact angle for samples $1-4$ in Table 1 are between $28.3^{\circ}$ and $62.3^{\circ}$ indicating the $\mathrm{Cu}$ treated surfaces are more hydrophilic than the unmodified PET reference sample. Examination of the S4 C1s high resolution spectrum in Figure 1 reveals it is profoundly different in shape to that of the PET reference sample. The C1s high resolution spectrum in Figure 1 for S4 suggests, for this sample treatment, the carbon is more graphitic in nature with very different $\mathrm{C}-\mathrm{O} / \mathrm{C}=\mathrm{O}$ bonding to $\mathrm{PET}$. Analysis of the $\mathrm{XPS} \mathrm{Cu} 2 \mathrm{p}_{3 / 2}$ high resolution spectra for samples $\mathrm{S} 1-\mathrm{S} 4$ reveals that the $\mathrm{Cu}$ is deposited primarily as $\mathrm{CuO}$ as a $\mathrm{Cu}$ shake up satellite is observed at a binding energy of $\sim 943 \mathrm{eV}$ which is diagnostic of $\mathrm{Cu}^{2+}$ species. 
For samples S5, S6 and S7, having undergone a Ti pre-treatment, the Ti concentration is low at only $1-4$ at. \% (see Table 1 ). Analysis of the XPS Ti2 $\mathrm{p}_{3 / 2}$ high resolution spectra reveals that the $\mathrm{Ti}$ is deposited as $\mathrm{TiO}_{2}$ (the $\mathrm{Ti}_{2} \mathrm{p}_{3 / 2}$ peak is observed at a binding energy of $458.8 \mathrm{eV}$ ). Examination of the $\mathrm{C} 1 \mathrm{~s}$ high resolution spectrum in Figure 1 for sample S5 shows the C1s peak-shape to be comparable to that of the PET reference samples indicating the S5 sample surface has retained its PET structure and there is probably an island-like distribution of $\mathrm{TiO}_{2}$ on the substrate. The water contact angles measured for S5, S6 and S7 ranges from $50.4^{\circ}$ to $64.7^{\circ}$ which is less than that observed for the PET reference sample, suggesting the Ti pretreatment has made the samples surface more hydrophilic but that it retains some of its PET character. Surface modification accomplished by the inclusion of a small concentration of a transition metal oxide, as observed in this case, is known to beneficially affect coating adhesion.

ToF-SIMS analysis, in the positive ion mode, of the PET reference film contains peaks diagnostic of PET at 104, 149, 191 and 193 u, these peaks are observed in Figure 2a for the PET reference sample. In the mass spectrum in Figure $2 b$ for the $S 2$ sample, the mass spectrum is dominated by copper and copper-oxygen peaks (with some common hydrocarbon peaks) while the mass peaks characteristic of PET are no longer observed. This suggests that the $\mathrm{CuO}$ surface layer is sufficiently thick that it spans the SIMS analysis depth ( $1 \mathrm{~nm})$. Similar ToF-SIMS results were obtained for all $4 \mathrm{Cu}$ modified PET surfaces regardless of power source or process parameter. The mass spectra of the Ti pre-treated surfaces show peaks diagnostic of PET in Figure 2c for the Ti pre-treated S6 sample, consistent with the XPS results. Ti and titanium-oxygen peaks are also observed in the S6 spectrum in Figure 2c but are very weak, compared to the intense peaks observed for $\mathrm{Cu}$ and PET in Figures $2 \mathrm{~b}$ and $2 \mathrm{a}$ respectively, such that they cannot be observed on the scale employed. The ToF-SIMS results are in good agreement with the quantitative data obtained from XPS in suggesting that the Cu pre-treatment 
resulted in the formation of a distinct thin film layer while the Ti pre-treatment resulted in a small degree of $\mathrm{TiO}_{2}$ incorporation into the PET surface.

Results obtained from parallel angle resolved (PARXPS) analyses revealed that the $\mathrm{Cu}$ concentration in the S3 and S4 samples progressively increases in intensity when moving from the surface towards the bulk sensitive angles. This indicates that for the HIPIMS samples the Cu may be present to depths beyond the analysis depth of an Al Ka X-ray source i.e. beyond 5$6 \mathrm{~nm}$. Consequently, XPS depth profiles were recorded, the results of the depth profile for sample S4 are shown in Figure 3. At the outer surface of the depth profile in Figure 3, it can be seen that the $\mathrm{C}, \mathrm{Cu}$ and $\mathrm{O}$ have similar intensities. Once etching commences for the depth profile, the $\mathrm{O}$ concentration remains constant, but there is a sharp decrease in the $\mathrm{C}$ concentration and a concomitant increase in the $\mathrm{Cu}$ signal intensity. The $\mathrm{C} 1 \mathrm{~s}$ peak from the outer surface of sample S4 (shown in Figure 1) can now be understood to be adventitious carbon contamination. In the near surface region of the depth profile, it is clear that there is a layer in which the $\mathrm{Cu}: \mathrm{O}$ ratio remains relatively constant at $1: 1$ with the $\mathrm{C}$ concentration being $<4$ at.\%. Throughout the depth of this layer, the $\mathrm{Cu} 2 \mathrm{p}_{3 / 2}$ peak continues to exhibit the strong shake up satellite at $943 \mathrm{eV}$ indicating that this layer is composed of $\mathrm{CuO}$, as expected from the $\mathrm{Cu}: \mathrm{O}$ stoichiometric ratio. The carbon peak in this region, is composed predominantly of graphitic carbon. As the depth profile progresses beyond the CuO layer, the data shows that the underlying material is composed of $\mathrm{C}$ and $\mathrm{O}$. The high $\mathrm{C}$ and low $\mathrm{O}$ concentrations observed at these depths, compared to that of native PET, is almost certainly the result of ion beam degradation of PET. All of the Cu pre-treatment samples showed the formation of a discrete CuO layer, with layer thickness varying between 1 and $7 \mathrm{~nm}$. With regard to relative thicknesses, the CuO layers deposited by HIPIMS were thicker than those deposited by p-DC and for both power modes, the unbalanced magnetic configuration gave rise to greater $\mathrm{CuO}$ film thicknesses than the unbalanced mode. 
PARXPS and XPS depth profile data for the Ti pre-treated samples showed the presence of only small amounts of $\mathrm{Ti}$ oxide in the surface region, which decreased in concentration with depth, this is consistent with a small quantity of $\mathrm{TiO}_{2}$ being incorporated into the outer surface of the PET. A more comprehensive series of XPS analyses for this work can be found in [20], in addition AFM topography data for samples S1 - S7 are included in [20].

The results presented here indicate there are significant differences in the chemical composition of PET surfaces plasma pre-treated using either a p-DC or HIPIMS oxygen-containing glow discharge. All the processing parameters investigated in this study appear to have a significant influence on the evolution of the surface chemistry and the resulting physical properties of the surfaces produced.

\section{Conclusions}

The chemical composition and film thicknesses of a series of pre-treated PET samples have been comprehensively characterized using XPS, ToF-SIMS, XPS depth profiling and static drop water contact angles. The results of this study suggest that the chemical composition and surface properties of plasma pre-treated PET surfaces, treated by reactive sputtering of $\mathrm{Cu}$ or $\mathrm{Ti}$ targets in a mixed $\mathrm{Ar}-\mathrm{O}_{2}$ atmosphere employing p-DC or HIPIMS power depends significantly on the processing parameters employed: sputter target material, magnetic array type and power supply technology. These results demonstrate that the sputter target material is vitally important as it defines the amount of metal deposited/implanted on the web surface. The use of the $\mathrm{Cu}$ target resulted in the formation of a discrete CuO film onto PET surface, with a thickness varying between 1 and $7 \mathrm{~nm}$, depending on the power mode and magnetic configuration employed. The use of the $\mathrm{Ti}$ target resulted in a small amount of $\mathrm{TiO}_{2}$ deposited, this being present probably as an island-like distribution on the PET surface. Most importantly, the different targets and processing configurations explored show that these pre-treatment 
processes lead to more hydrophilic surfaces and hence promote adhesion of subsequent layers on the polymer surface. They also offer a flexible means to achieve a wide range of surface chemistries and hence properties that can be tailored according to the needs of different applications.

\section{Acknowledgements.}

The authors thank Mr Ron Logan for the contact angle measurements. 


\section{References.}

[1] R. Ludwig, R. Kukla, E. Josephson, Proceedings of the IEEE 93 (2005) 1483.

[2] J.E.E. Baglin, Nuclear Inst. and Methods in Physics Research, B 65 (1992) 119.

[3] R.W. Burger, L.J. Gerenser, 34th Annual Technical Conference, Society of Vacuum Coaters, 1991 , p. 162.

[4] F.D. Egitto, L.J. Matienzo, 36th Annual Technical Conference, Society of Vacuum Coaters, 1993, p. 10.

[5] E. Finson, S. Kaplan, L. Wood, 38th Annual Technical Conference, Society of Vacuum Coaters, 1995, p. 52.

[6] M. Geisler, G. Hoffmann, R. Ludwig, G. Steiniger, Surface and Coatings Technology 177178 (2004) 735.

[7] R. Ludwig, R. Kukla, H.J. Lotz, G. Hoffmann, G. Steiniger, P. Sauer, L. Josephson, 48th Annual Technical Conference, Society of Vacuum Coaters, 2005, p. 229.

[8] H. Morgner, R. Rank, J. Reschke, N. Schiller, 42nd Annual Technical Conference, Society of Vacuum Coaters, 1999, p. 460.

[9] S.A. Pirzada, A. Yializis, W. Decker, R.E. Ellwanger, 42nd Annual Technical Conference, Society of Vacuum Coaters, 1999, p. 301.

[10] A. Yializis, M.G. Mikhael, R.E. Ellwanger, 42nd Annual Technical Conference, Society of Vacuum Coaters, 1999, p. 469.

[11] R. Rank, T. Wuensche, M. Fahland, C. Charton, N. Schiller, 47th Annual Technical Conference, Society of Vacuum Coaters, 2004, p. 632.

[12] W. Schönberger, G. Gerlach, M. Fahland, P. Munzert, U. Schulz, R. Thielsch, R. Kleinhempel, Surface and Coatings Technology In Press, Accepted Manuscript (2011).

[13] G. Este, W.D. Westwood, Journal of Vacuum Science \& Technology A: Vacuum, Surfaces, and Films 6 (1988) 1845.

[14] V. Kouznetsov, Canada Patent No. CA22841811998.

[15] J. Bohlmark, M. Lattemann, J.T. Gudmundsson, A.P. Ehiasarian, Y. Aranda Gonzalvo, N. Brenning, U. Helmersson, Thin Solid Films 515 (2006) 1522.

[16] A.P. Ehiasarian, Y.A. Gonzalvo, T.D. Whitmore, Plasma Processes and Polymers 4 (2007).

[17] J. Lin, J.J. Moore, W.D. Sproul, B. Mishra, J.A. Rees, Z. Wu, R. Chistyakov, B. Abraham, Surface and Coatings Technology 203 (2009) 3676.

[18] E.Vassallo, A.Cremona, F.Ghezzi, D.Ricci, Vacuum 84 (2010) 902

[19] In High Resolution XPS of Organic Polymers - The Scienta ESCA300 Database, G Beamson and D Briggs, John Wiley \& Sons publishers. (1992) 174-175.

[20] M.Audronis, S.J.Hinder, P.Mack, V.Bellido-Gonzalez, D.Bussey, A.Matthews, M.A.Baker, Thin Solid Films 520 (2011) 1564. 


\begin{tabular}{|l|l|l|l|l|l|l|l|l|}
\hline \multicolumn{2}{|l|}{ Sample } & $\begin{array}{l}\text { Sputter } \\
\text { Target }\end{array}$ & Magnetic & Power \\
Technology & $\begin{array}{l}\text { C1s } \\
\text { (At.\%) }\end{array}$ & $\begin{array}{l}\text { O1s } \\
\text { (At.\%) }\end{array}$ & $\begin{array}{l}\text { Cu2p } \\
\text { (At.\%) }\end{array}$ & $\begin{array}{l}\text { Ti2p } \\
\text { (At.\%) }\end{array}$ & $\begin{array}{l}\text { Contact } \\
\text { Angle } \\
\left({ }^{\circ}\right.\end{array}$ \\
\hline PET & & & & 67.0 & 33.0 & & & 74.3 \\
\hline S1 & $\mathrm{Cu}$ & Balanced & p-DC & 28.9 & 37.5 & 33.0 & & 62.0 \\
\hline S2 & $\mathrm{Cu}$ & Unbalanced & p-DC & 31.0 & 37.9 & 30.8 & & 62.3 \\
\hline S3 & $\mathrm{Cu}$ & Balanced & HIPIMS & 27.1 & 37.6 & 34.9 & & 48.8 \\
\hline S4 & $\mathrm{Cu}$ & Unbalanced & HIPIMS & 30.2 & 36.2 & 33.3 & & 28.3 \\
\hline S5 & $\mathrm{Ti}$ & Balanced & p-DC & 59.2 & 35.2 & - & 4.0 & 64.7 \\
\hline S6 & $\mathrm{Ti}$ & Balanced & HIPIMS & 59.8 & 35.4 & - & 1.9 & 58.2 \\
\hline S7 & $\mathrm{Ti}$ & low (RF) & HIPIMS & 64.4 & 33.1 & - & 0.9 & 50.4 \\
\hline
\end{tabular}

Table 1. Process variables, XPS elemental composition and water contact angles of the plasma treated PET samples. 


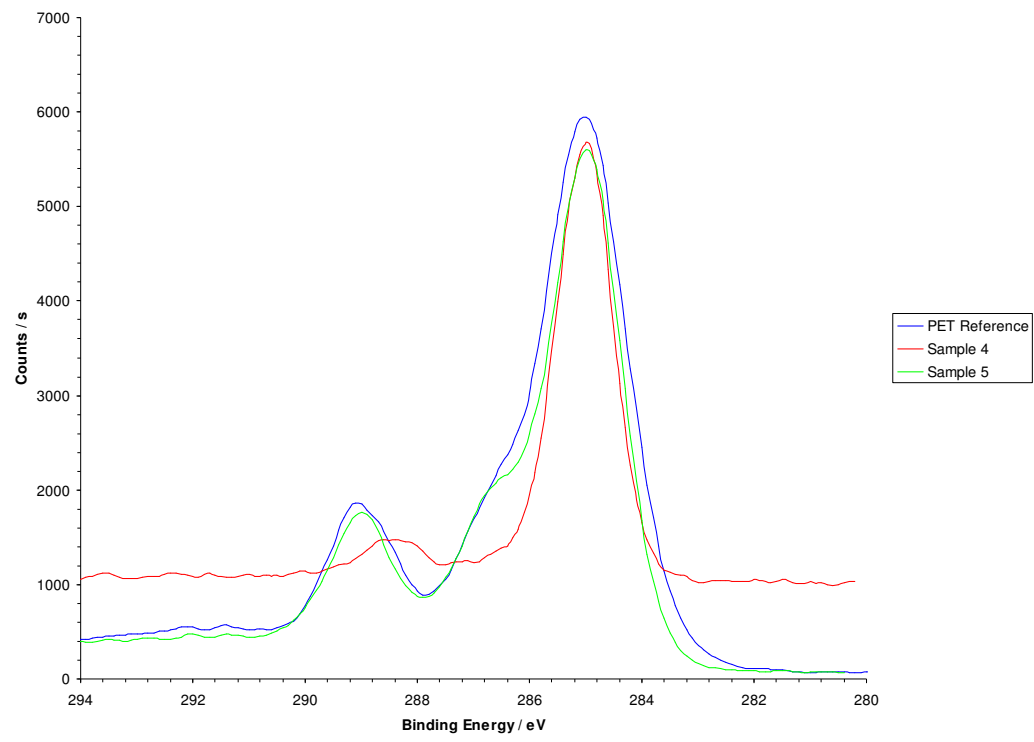

Figure 1. XPS C1s high resolution spectra for the PET reference, S4 and S5 samples. 
a)

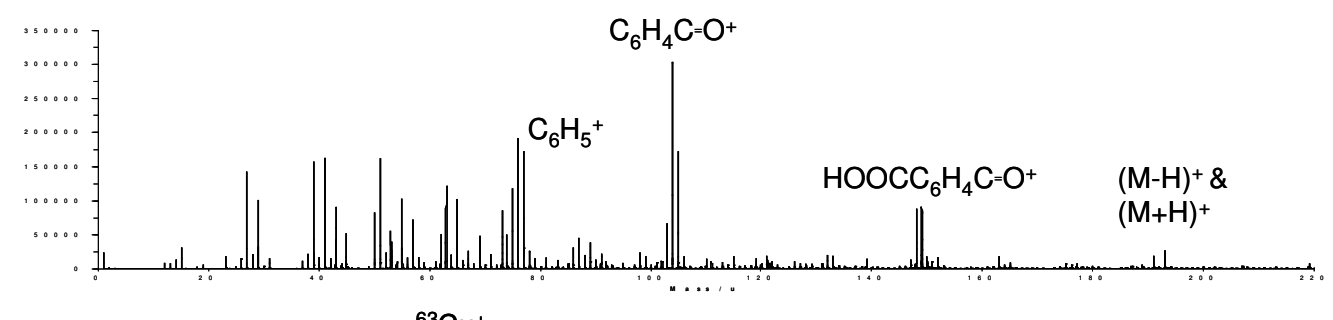

b)

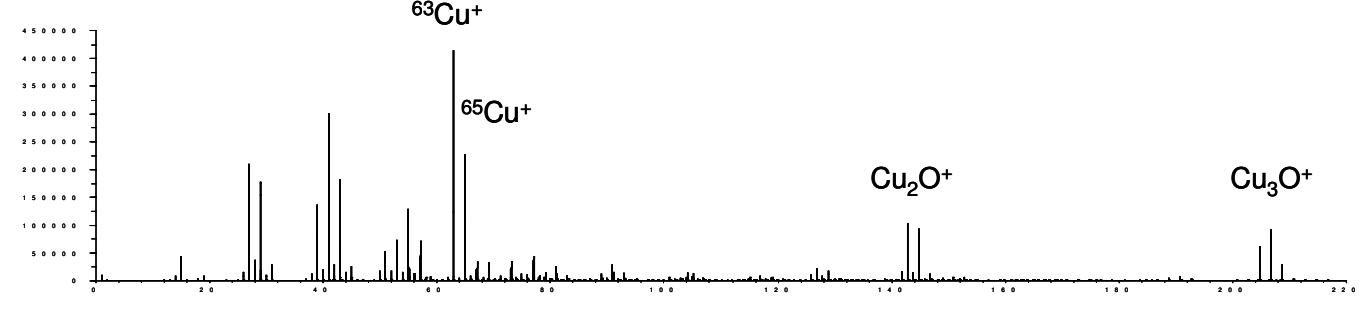

c)

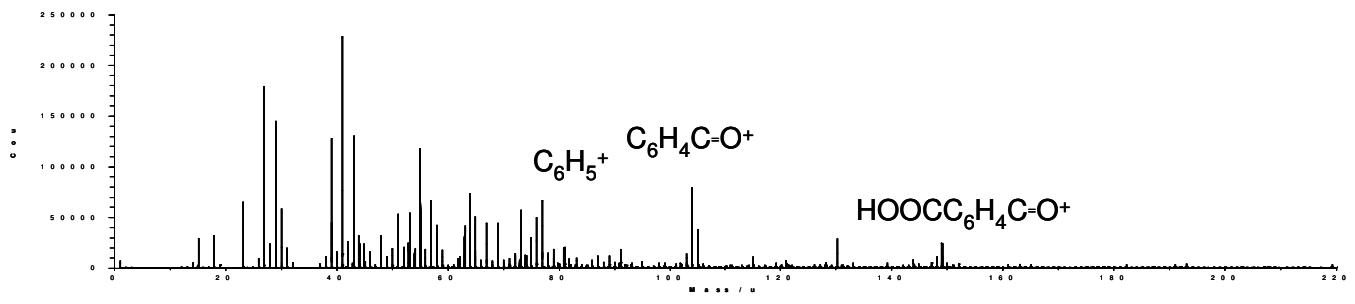

Figure 2. ToF-SIMS positive ion mass spectra of a) the PET reference, b) sample S2 and c) sample S6. 


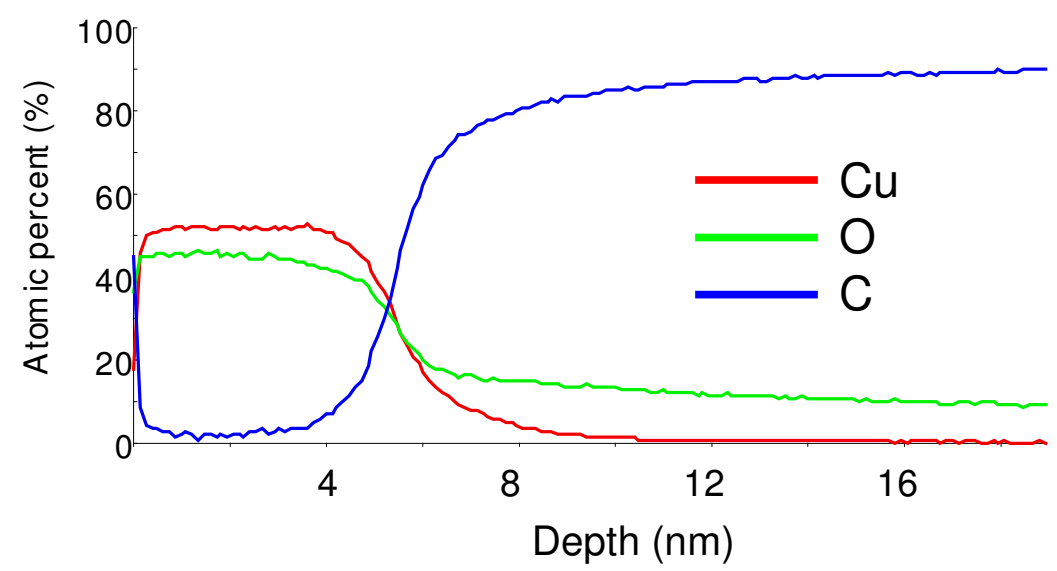

Figure 3. XPS depth profile of sample S4 after HIPIMS Cu pre-treatment of PET. 